\title{
An Efficient Synthesis of Tetrodotoxin
}

David B. Konrad, ${ }^{a, t, \# ~ K l a u s-P e t e r ~ R u ̈ h m a n n, ~}{ }^{b}$ Hiroyasu Ando, ${ }^{c}$ Belinda E. Hetzler, ${ }^{b}$ Bryan S. Matsuura,,$^{\mathrm{b}, \#, \star}$ and Dirk Traunera,b,*

aDepartment of Chemistry, Ludwig-Maximilians-Universität München, Butenandtstr. 5-13, 81377 Munich, Germany

bDepartment of Chemistry, New York University, 100 Washington Square East, New York 10003, United States

'Graduate School of Infection Control Sciences and Kitasato Institute for Life Sciences, Kitasato University, Tokyo, Japan

†Current address: Department of Pharmacy, Ludwig-Maximilians-Universität München, Butenandtstr. 5-13, 81377 Munich, Germany

"Contributed equally to this work

*To whom the correspondence should be addressed

\section{Abstract}

Tetrodotoxin (TTX) is an indispensable probe in neuroscience, a biosynthetic and ecological enigma, and one of the most celebrated targets of synthetic chemistry. Here, we present a stereoselective synthesis of TTX that proceeds in 22 steps starting from a readily available glucose derivative. The central cyclohexane ring of TTX and its $\alpha$-tertiary amine moiety was established via the intramolecular 1,3-dipolar cycloaddition of a nitrile oxide, followed by alkynyl addition to the resultant isoxazoline. After some carefully chosen protecting group manipulations, a ruthenium-catalyzed hydroxylactonization set the stage for the formation of its dioxa-adamantane core. Installation of the guanidine, oxidation of a primary alcohol, and late-stage epimerization of the resultant aldehyde gave a mixture of TTX and anhydro TTX. Our synthesis represents one of the most effective of TTX reported to date and could give ready access to biologically active derivatives. 


\section{Introduction}

Tetrodotoxin (TTX) is a neurotoxic natural product that has inspired and empowered chemists and biologists for more than a century. ${ }^{1-3}$ As a selective blocker of voltage-gated sodium channels, it has played a crucial role in the elucidation of the action potential, and it is still routinely used to silence excitable cells in neural systems. Its widespread occurrence in aquatic animals, such as pufferfish, starfish, sea snails, octopi, and even in newts, has prompted intense investigations into its true biological producers, its biosynthesis, and its ecological role. It is now clear that TTX is synthesized by symbiotic bacteria and accumulated by their metazoan hosts as a defense against predators. ${ }^{4}$ Its toxicology and therapeutic utility in humans has been studied for decades and is still a matter of ongoing research. ${ }^{5}$

As a synthetic target, TTX has been celebrated for the sheer intellectual challenge it provides and for the opportunity to demonstrate methodological and strategic advances. Its simple carbon framework, consisting of a cyclohexane ring with $\mathrm{C} 1$ and $\mathrm{C} 2$ side chains, stands in stark contrast to the dense network of polar functional groups that adorn it. Two hydroxy groups in a syn relationship engage a carboxylate as an ortho acid to form the signature dioxaadamantane core of TTX, which is fused to a cyclic guanidine via an $\alpha$ tertiary amine. One primary, two secondary, and a tertiary hydroxy group, as well as an $\mathrm{N}, \mathrm{O}$-hemiacetal, contribute further to the structural complexity of the molecule, which features four rings and nine contiguous stereocenters.

The first total synthesis of TTX, in racemic form, by Kishi and Fukuyama in 1972 stands as a landmark achievement in organic synthesis that, at the time, seemed hard to surpass. ${ }^{6}$ After a pause of more than 30 years, Isobe published the first asymmetric synthesis in 2003. ${ }^{7}$ This was followed shortly by the DuBois' asymmetric synthesis (2003), ${ }^{8}$ a second Isobe approach (2004), ${ }^{9,10}$ as well as a racemic and two asymmetric syntheses by Sato (2005, 2008 and 2010, respectively). ${ }^{11-13}$ In 2017 and 2020, Fukuyama, Yokoshima, and colleagues revisited the molecule and published two distinct 
asymmetric routes to TTX.14,15 In addition, several studies have been published that intercept late-stage intermediates of the previous syntheses e.g. by Alonso (2010), ${ }^{16,17}$ Ciufolini (2015), ${ }^{18-20}$ and Hudlicky (2018). ${ }^{21}$ Other approaches toward the molecule have been outlined. ${ }^{19,22-32}$

\section{Synthetic Design}

An analysis of previous syntheses revealed several common features that informed our approach: 1) The cyclohexane core is either incorporated in the starting material, or is formed early, and then oxygens are added using epoxidations, dihydroxylations, or allylic oxidations of strategically placed alkenes. 2) The $\alpha$-tertiary amine (ATA) on C8a is established by a $\mathrm{C}-\mathrm{N}$ bond formation, which needs to overcome considerable steric hinderance. Several methods have been implemented to address this, such as intramolecular nitrogen transfer (sigmatropic rearrangement, aza-conjugate addition, nitrene rearrangement/insertion) and intermolecular $\mathrm{S}_{\mathrm{N}} 1$-type nucleophilic substitution. 3) The dioxaadamantane is always formed from a lactone with a stereogenic a-hydroxy group. Careful orchestration of the sequence is needed to adjust the oxidation state C10, form the lactone, and set the labile C9 stereocenter. 4) Every synthesis introduces the guanidine at a late stage $e^{7,33}$ and utilizes protecting groups amenable to global deprotection in the final step.

Our synthetic analysis was guided by an attempt to link the formation of the cyclohexane core with the establishment of the ATA as closely as possible, in contrast to previous total syntheses where these strategic key steps were largely independent. ${ }^{17}$ To this end, we needed to establish a linear precursor that contained all the oxygenation of the TTX skeleton, which would be conjoined by a ring-forming reaction. This would be followed by the ATA formation through $C-C$ bond formation, to introduce the $\mathrm{C} 2$ fragment that would eventually be incorporated into the dioxaadamantane. Finally, we needed to develop a method to oxidize and lactonize this $\mathrm{C} 2$ fragment in a highly efficient manner. 


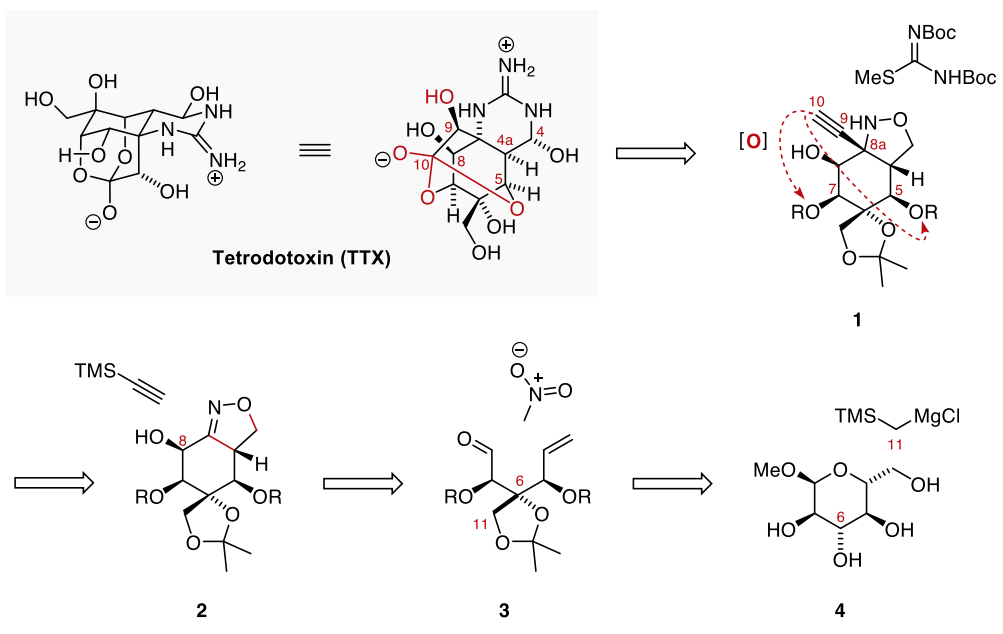

Figure 1. Retrosynthetic analysis and synthetic design.

Our retrosynthetic analysis is summarized in Figure 1. Although not all compounds shown therein were defined in such detail at the outset of our study, it captures the essence of our synthetic plan. We reasoned that we could trace TTX back from an oxidation of an alkynyl isoxazolidine of type $\mathbf{1}$, which would stem from bicyclic isoxazoline $\mathbf{2}$, the product of an intramolecular 1,3-dipolar cycloaddition. Nitromethane would serve as a key linchpin in the assembly of $\mathbf{2}$, reacting intermolecularly with aldehyde $\mathbf{3}$, via a Henry reaction, followed by a dehydration to generate a reactive nitrile oxide that would close the central cyclohexane ring with a $(3+2)$ cycloaddition. We have previously developed an asymmetric synthesis of unsaturated aldehydes similar to $\mathbf{3}$ via a Kiyooka aldol reaction and used it toward kweichowenol A, a polyoxygenated cyclohexene isolated from the plant Uvaria kweichowensis. ${ }^{34}$ Although an analogous route gave the aldehyde $\mathbf{3}$ in sufficient quantities to procced with the synthesis of TTX, we found it more practical and economical to start from the glucose-derived building block 4 . All the carbons of glucose and two of its stereocenters would be retained over the course of the synthesis, making this an attractive starting material. 


\section{Synthesis of the Carbon Skeleton}

Our actual synthesis started with the known C- 6 building block 5 , which is available in 5 steps from methyl $\alpha$-D-glucopyranoside on a decagram scale and was previously used by Sato and colleagues in their approach to TTX. ${ }^{13}$ Regioselective reductive cleavage of the benzylidene acetal placed a benzyl ether at C5, yielding 6 (Figure 2). A highly diastereoselective dihydroxylation then installed the tertiary alcohol with the correct absolute configuration a $\mathrm{C} 6$, as well as the $\mathrm{C} 11$ primary alcohol of TTX, providing 7 in excellent yield. ${ }^{35}$ Protection of the vicinal diol as the acetonide, followed by Appel reaction gave the primary iodide 8 . Using conditions developed by Soengas and Silva, 8 underwent reductive cleavage upon treatment with tert-butyl lithium at low temperature, to yield $\delta, \varepsilon$-unsaturated aldehyde (12, Figure 3 ), which underwent a Henry reaction in situ upon addition of $\mathrm{MeNO}_{2}$. This afforded nitroalcohols $\mathbf{9 a , b}$ as a separable mixture of diastereomers. ${ }^{36}$
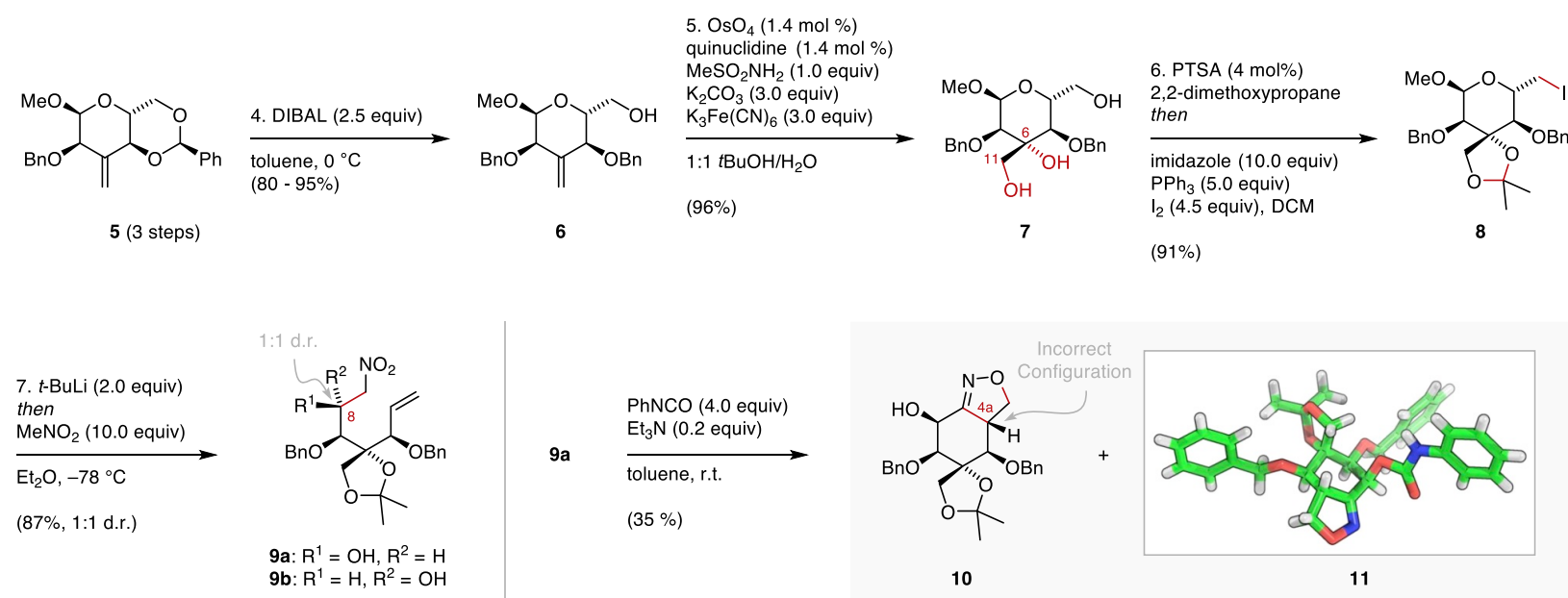

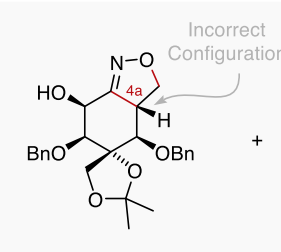

10

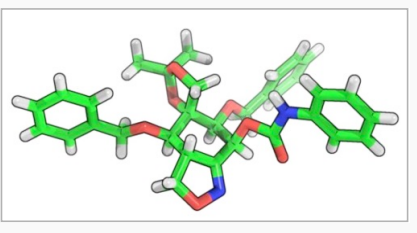

11

Figure 2. Opening sequence and initial attempts to form the carbocyclic core.

With nitroalcohols $\mathbf{9 a , b}$ in hand, we were ready to attempt the key nitrile oxide cycloaddition to form the core cyclohexane of tetrodotoxin. Treatment of diastereomer 9a with phenylisocyanate and triethylamine triggered a dehydrative nitrile oxide cycloaddition, yielding isooxazoline $\mathbf{1 0}$ in moderate yield as a single diastereomer. ${ }^{37} \mathrm{An}$ 
X-ray structure of the corresponding $N$-phenyl carbamate 11 confirmed the configurations of the $\mathrm{C} 8$ and $\mathrm{C} 4$ a stereocenters. Although the $\mathrm{C} 4{ }_{a}$ stereocenter was inverted with respect to TTX, we reasoned that this isoxazoline diastereomer would be more accessible to nucleophiles for the formation of the hindered $\alpha$-tertiary amine. ${ }^{38}$ Exposing diastereomer $9 b$ to the same reaction conditions only resulted in decomposition.
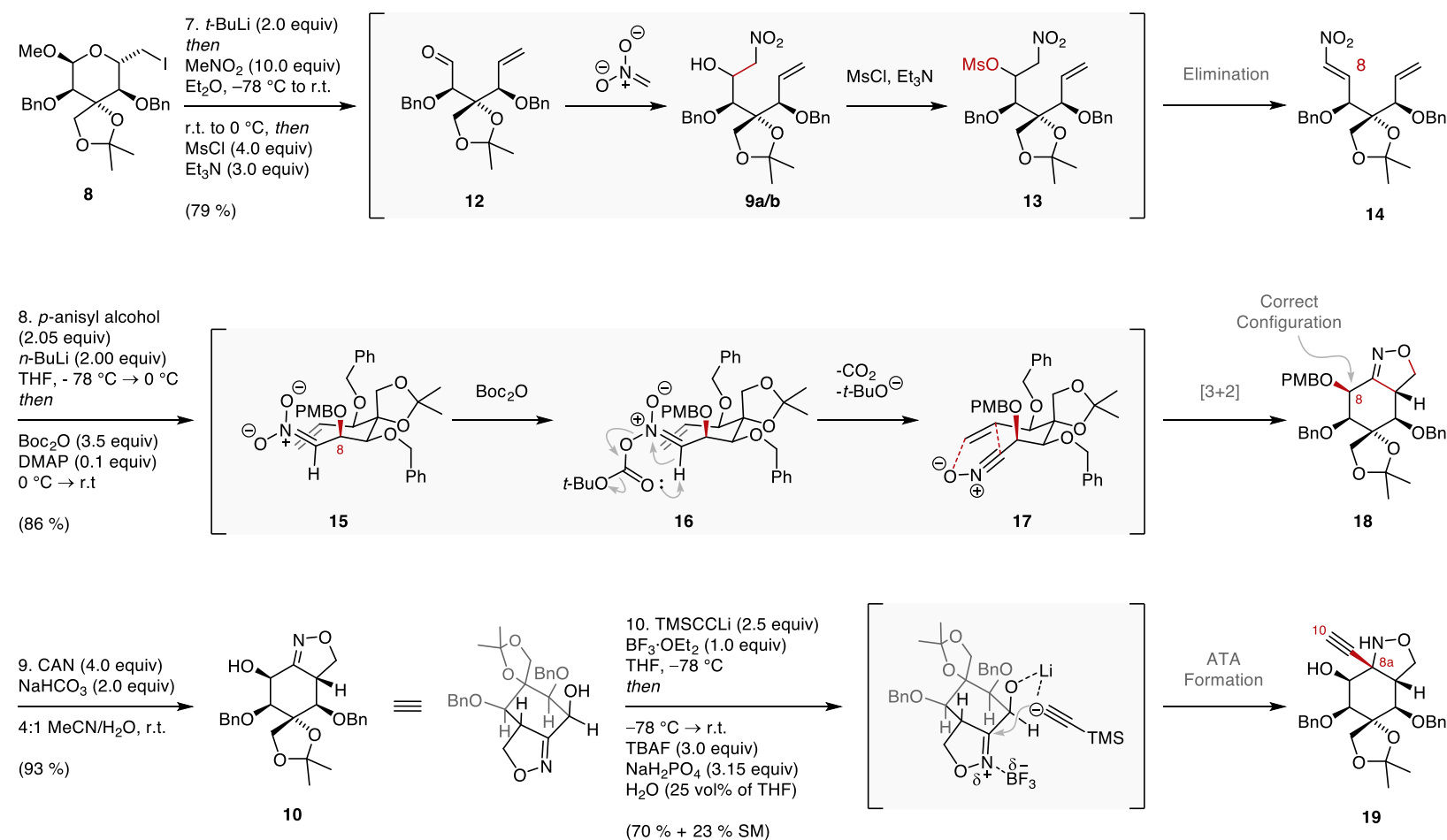

Figure 3. Development of a diastereoselective route to the cyclohexane core and installation of the ATA.

The poor diastereoselectivity of the Henry reaction and low yields of the cycloaddition severely hampered material throughput. Moving forward, we needed to develop a strategy that would selectively install a stereocenter at C8 - ideally with a protected hydroxyl - which is critical in the ensuing cycloaddition. We reasoned that this could be accomplished via a dehydration to the corresponding nitroalkene followed by a conjugate addition with an appropriate $O$-nucleophile. This was realized by subjecting 8 to the same reductive fragmentation/Henry reaction, followed by in situ dehydration, 
which yielded nitroalkene 14 exclusively as the E-isomer. 14 underwent an oxa-Michael addition upon treatment with the lithiated alkoxide of $p$-anisyl alcohol, presumably resulting in nitronate anion 15. This intermediate could be intercepted with Boc-anhydride (via 16) to trigger the formation of intermediate nitrile oxide 17 and subsequent 1,3-dipolar cycloaddition affording isoxazoline $\mathbf{1 8}$ in high yield and on a decagram scale. ${ }^{39}$ Notably, this reaction cascade was exquisitely diastereoselective, affording the central cyclohexane core of TTX with the correct configuration at $\mathrm{C} 8$, albeit with the wrong configuration at $\mathrm{C} 4$. Following deprotection of the $\mathrm{PMB}$ group with $\mathrm{CAN}, 10$ was subjected to lithiated TMS-acetylide and $\mathrm{BF}_{3} \cdot \mathrm{OEt}_{2}$, which underwent addition from the convex side of the bicyclic isoxazoline, directed by the proximal alcohol. ${ }^{40}$ Following cleavage of the TMS group upon workup, isoxazolidine alkyne 19 was isolated as a single diastereomer, which possesses all the skeletal carbons of TTX and 5 out of its 9 stereocenters with the correct absolute configuration. Notably, the presence of a free hydroxy group, which is presumably deprotonated under the conditions of the reaction, was found to be crucial for the success of the nucleophilic addition to the oxime ether moiety. The lithiated TMSacetylide was singularly effective, as more highly functionalized C2 synthons were found to be unreactive.

\section{Oxidative elaboration of the side chain and completion of the synthesis}

Our next goal was the oxidative elaboration of the triple bond to introduce the stereogenic $\mathrm{C} 9$ alcohol and the $\mathrm{C} 10$ lactone that would ultimately engage in the formation of the signature ortho-acid of TTX. To this end, we needed to establish a protecting group scheme that would also be compatible with the subsequent introduction of the guanidine moiety and the projected end game. Exposure of 19 to $\mathrm{Boc}_{2} \mathrm{O}$ gave 20, which features both a tert-butyl carbonate and carbamate moiety. The next critical deprotection step required the simultaneous cleavage of the $\mathrm{C} 5$ and $\mathrm{C} 7$ benzyl ethers in the presence of the three other acid sensitive protecting groups, an alkyne and an isoxazolidine, both of which are sensitive to hydrogenation. After considerable experimentation, we found that the benzyl ethers could be cleanly removed using Pieber and Seeberger's recently 
introduced chromoselective photochemical debenzylation, a singularly effective protocol that afforded diol 21 in excellent yield. ${ }^{41}$ The Boc-group on the C8-alcohol and the use of $525 \mathrm{~nm}$ green LED as the irradiation source were key to this reaction's success. The use of either the unprotected alcohol or higher-energy blue light resulted in complete substrate decomposition. Following the debenzylation, a selective methanolysis of the carbonate gave a triol, which could be protected to bis-acetonide 22. At this stage, we elected to reduce the sensitive $\mathrm{N}$-Boc oxazolidine in 22. Using $\mathrm{Sml}_{2}$, the $\mathrm{N}-\mathrm{O}$ was cleaved, resulting in a primary alcohol in excellent yield, which was then protected as its silyl ether 23 (Figure 4).

The stage was now set for the next key step of our synthesis, the conversion of alkyne 23 to hydroxylactone 24 . To take full advantage of the steric environment provided by the proximal acetonide, we aimed at forming the $\mathrm{C} 10-\mathrm{O} 5$ bond first to yield a dihydropyran that could be then oxidized to the hydroxylactone in a stereoselective manner. Initially, we attempted to achieve this via a 6-endo-dig cyclization using gold or silver-based $\Pi$-acid catalysts, but this was thwarted by the substrate's propensity to undergo undesired 5-exo-dig cyclization.

Our solution to this problem was inspired by reports from Trost $^{42}$ and McDonald ${ }^{43}$ on the catalytic generation of metallovinylidene carbenes that render the alkyne terminus electrophilic. We found that 23 could be converted to dihydropyran 24, using $\mathrm{CpRu}\left(\mathrm{PPh}_{3}\right)_{2} \mathrm{Cl}$ as a cycloisomerization catalyst, in nearly quantitative yield on a $300 \mathrm{mg}$ scale. ${ }^{44}$ Pushing this finding even further, we postulated that the resultant dihydropyran could be converted to the key hydroxylactone 25 by transforming the cycloisomerization catalyst into an oxidant. ${ }^{45}$ This was achieved through by the addition of Oxone and a cosolvent mixture ${ }^{46}$ to the reaction mixture. Presumably, under these conditions, the catalyst is oxidized to $\mathrm{RuO}_{4}$, which in turn oxidized $\mathbf{2 4}$ to the desired hydroxylactone $\mathbf{2 5}$, with almost complete diastereoselectivity (Figure 4, bottom). This single reaction combines the $\mathrm{C} 10-\mathrm{O} 5$ bond formation with two-oxidation events, setting the C9 stereocenter in the processes, and had the added benefit of simplifying purification, since the cycloisomerization catalyst was copolar with 24 on silica. ${ }^{47}$ To the best of our 
knowledge, both the bridge-forming cycloisomerization reaction and transformation of $\mathrm{CpRu}\left(\mathrm{PPh}_{3}\right)_{2} \mathrm{Cl}$ to a catalytic oxidant are unprecedented.

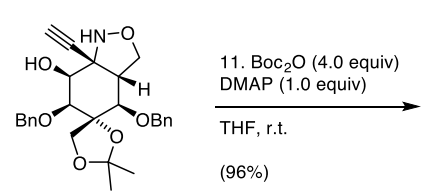

19

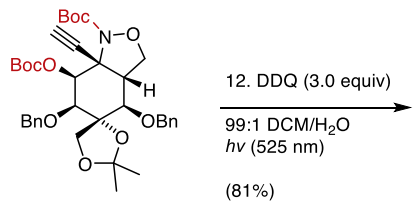

20

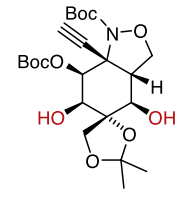

21

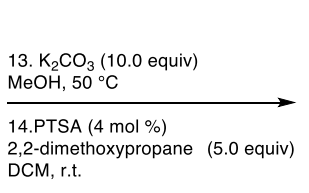

(98\%)

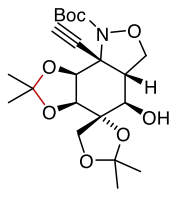

22
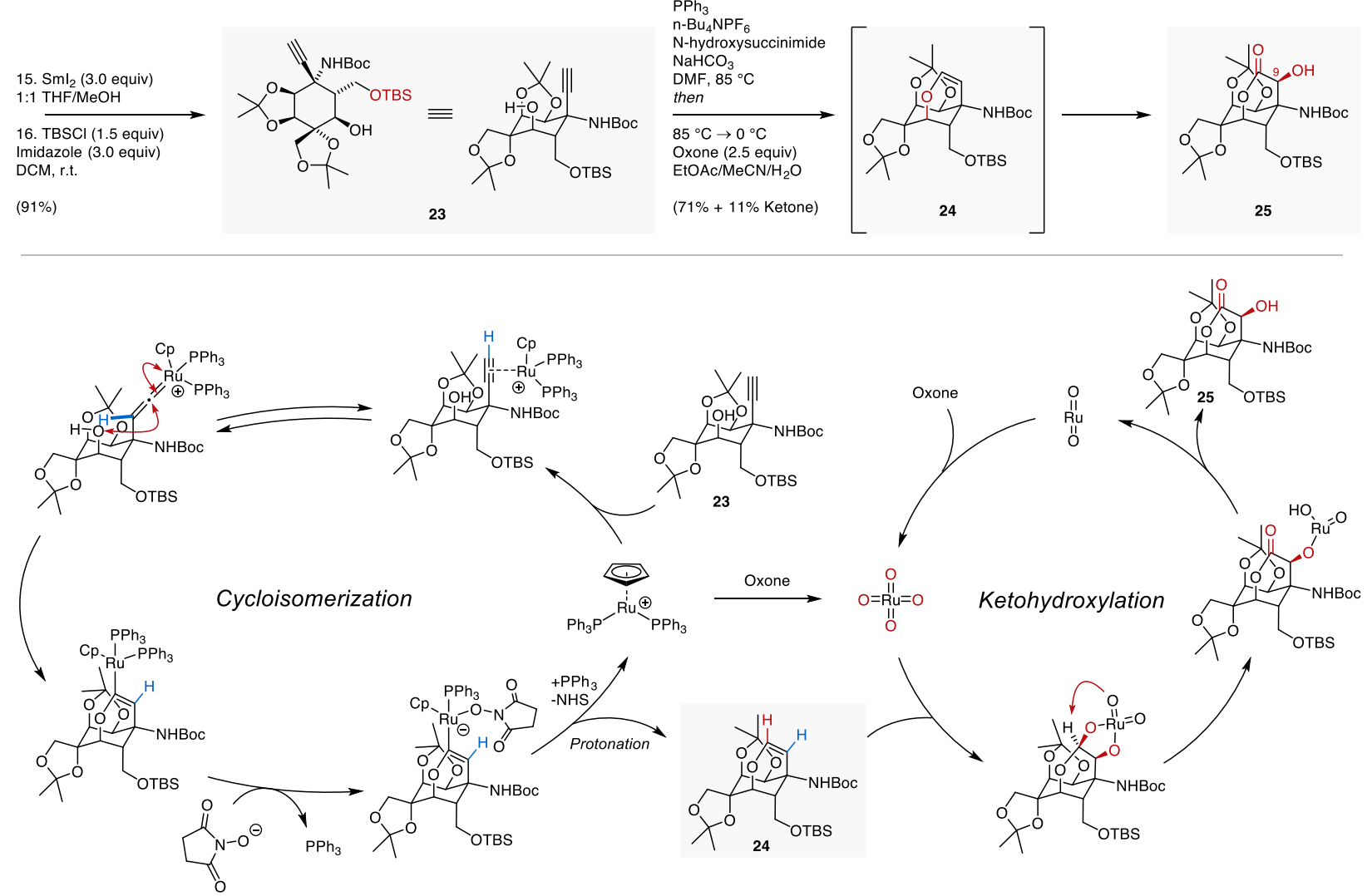

Figure 4. Continuation of the synthesis and proposed mechanism for the key hydroxylactonization.

Having found a satisfying solution for the hydroxy lactone problem, we decided to tackle the guanidine moiety next. Removal of the N-Boc protecting group with TMS triflate, followed by cleavage of the silyl ethers, one of which was transient, gave amino diol 26. This compound was protected in situ as the bis-trimethyl silyl ether and then underwent 
clean guanylation under Kishi's conditions to compound 27, which features all of the atoms of TTX (Figure 5).

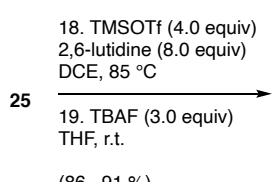

$(86-91 \%)$

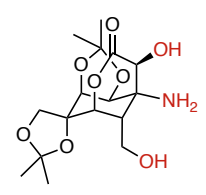

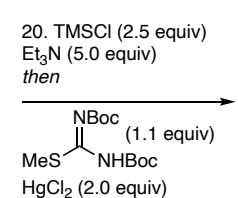

DMF, r.t.

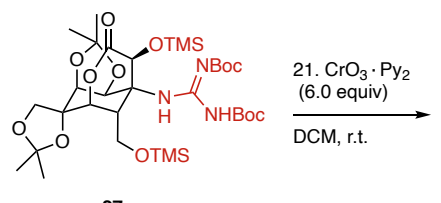

27

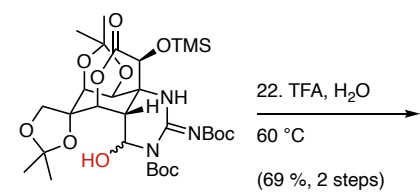

28

$(92 \%)$

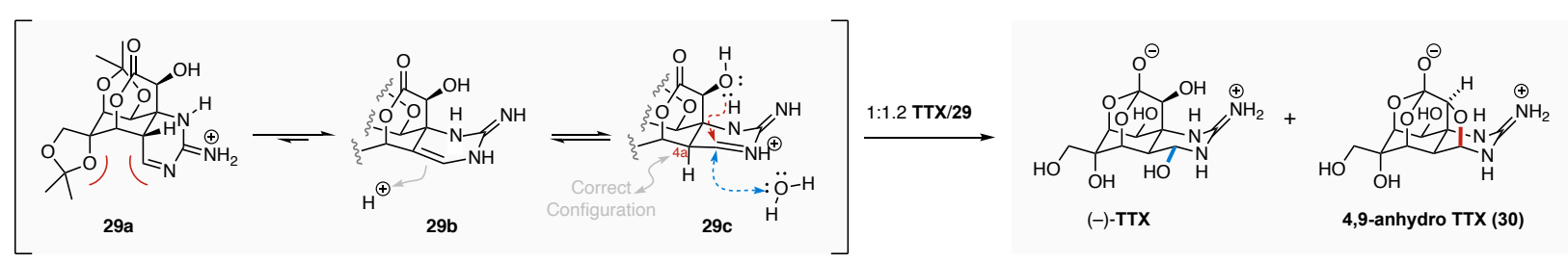

Figure 5. Completion of the synthesis.

In the last phase of our synthesis, we needed to address two final obstacles: the oxidation of the primary silyl ether and the epimerization of the C4a stereocenter. A footnote in Kishi's seminal publication suggested the latter presented a potential liability due to the propensity of the $\mathrm{C} 5-\mathrm{O}$ bond to undergo facile elimination, and all prior approaches had set this stereocenter earlier in their respective syntheses. ${ }^{6}$ Nevertheless, we continued by treating bis trimethyl silyl ether $\mathbf{2 7}$ with Collins' reagent, which effected selective deprotection and oxidation of the primary alcohol, resulting in $\mathbf{2 8}$ as a mixture of aminal isomers. Note that this transformation could not have been performed with a tertbutyl dimethyl silyl ether in place, in other words, with a guanidinylated derivative of compound 27. Crude $\mathbf{2 8}$ was then dissolved in $25 \%$ aqueous trifluoracetic acid and stirred at room temperature overnight. Fortuitously, this effected the desired deprotections, epimerization and cyclizations and gave a 1:1.2 mixture of TTX and 4,9-anhydro TTX (30) in good overall yield. TTX and $\mathbf{3 0}$ are known to be in equilibrium with one another, favoring TTX, and they can be readily interconverted. ${ }^{7,48}$ They have been separated on an analytical scale. ${ }^{49}$ Interestingly, in our case, the thermodynamically less stable $\mathbf{3 0}$ was observed in unusually high proportions, which could be explained by the guanidine participating in the epimerization process. Intramolecular condensation of the N3-nitrogen 
with the C4a aldehyde would form a highly stabilized iminium cation $29 \mathrm{a}$, which could undergo elimination to enamine 29b. Tautomerization to the more thermodynamically favored iminium 29c, situates $\mathrm{C} 4 \mathrm{a}$ in close proximity to the C9-hydroxy group, which can attack at a rate that is kinetically competitive with solvolysis. We believe that the elimination and tautomerization reactions are likely driven by unfavorable syn-pentane interactions between the C6-oxygen and the C4a-iminium. However, given the multitude of transformations that are taking place in this final step, it is difficult, if not impossible, to pinpoint the exact sequence of events, which could take place in parallel and converge on TTX.

\section{Conclusion}

In summary, we have developed a stereoselective synthesis of tetrodotoxin whose efficiency results from the identification of several strategic disconnections. Specifically, our route showcases the power of the Huisgen cycloaddition for the construction of highly substituted cyclitols. For this purpose, the humble $\mathrm{C} 1$ synthon nitromethane performed spectacularly - involved in no fewer than 6-bond making and breaking events - relaying an oxygen to $\mathrm{C} 4$, and embedding its nitrogen and carbon into the $\alpha$-tertiary amine over the course of the synthesis. Although the isoxazoline $\mathbf{1 0}$ possessed the incorrect stereocenter at $\mathrm{C} 4 \mathrm{a}$, necessitating a late-stage epimerization, it guided the subsequent acetylide addition to install the correct configuration of the C8a $\alpha$-tertiary amine. While this late-stage epimerization carried some strategic risk, we had reason to believe that it would succeed based on the coherence of the dioxaadamantane core, which would render beta-eliminations or retro-aldol reactions reversible. Of course, we also had designs in place in case it failed, such as dehydration/hydration sequences. The alkynylation of $\mathbf{1 0}$ emphasizes the utility of oxime ethers as ATA-precursors but also highlights the need for more methodological development in this area. The Ru-catalyzed oxidative lactonization of alkyne 23 represents a notable advance in establishing the C9/C10 hydroxylactone and should have future applications in the synthesis of other natural products such as the ginkolides and quassinoids. ${ }^{50,51}$ Furthermore, this synthesis 
served as a proving ground for the chromoselective photochemical debenzylation and recognizes the value of implementing new technology and methods in highly complex settings.

Taken together, these strategic decisions resulted in one of the shortest and the most efficient syntheses of tetrodotoxin to date, accomplishing this goal in 22 steps. The route is scalable and can be adapted for the production of other scarce tetrodotoxin derivatives and unnatural analogs to better understand their biosynthesis, chemical ecology, and their potential as next-generation analgesics.

\section{Acknowledgements}

This paper is dedicated to Prof. Edda Gössinger in recognition of her seminal contributions to the development of 1,3-dipolar cycloadditions. We gratefully acknowledge financial support by the NSF (CHE-1900154). D.B.K. gratefully acknowledges funding by the Friedrich-Ebert-Stiftung through a doctoral scholarship. K.P.R. and B.E.H. are supported by an NYU MacCracken Fellowship. H. A. was supported by the JSPS Overseas Challenge Program for Young Researchers. The authors thank Ivica Žamarija, Alexander Novak, Caren Wanzke, Kuno Schwärzer, Edward Miller, Rebecca Bechtel, Nathalie Kurrle and Bilal Kicin for experimental assistance. 


\section{Literature References}

1. Chau, J. \& Ciufolini, M. A. The Chemical Synthesis of Tetrodoxin: An Ongoing Quest. Marine Drugs 9, 2046-2074 (2011).

2. Nishikawa, T. \& Isobe, M. Synthesis of Tetrodotoxin, a Classic but Still Fascinating Natural Product. The Chemical Record 13, 286-302 (2013).

3. Makarova, M., Rycek, L., Hajicek, J., Baidilov, D. \& Hudlicky, T. Tetrodotoxin: History, Biology, and Synthesis. Angewandte Chemie International Edition 58, 18338-18387 (2019).

4. Hanifin, C. T. The Chemical and Evolutionary Ecology of Tetrodotoxin (TTX) Toxicity in Terrestrial Vertebrates. Marine Drugs 8, 577-593 (2010).

5. Moczydlowski, E. G. The molecular mystique of tetrodotoxin. Toxicon 63, 165-183 (2013).

6. Kishi, Y. et al. Synthetic studies on tetrodotoxin and related compounds. IV. Stereospecific total syntheses of DL-tetrodotoxin. J. Am. Chem. Soc. 94, 9219-9221 (1972).

7. Ohyabu, N., Nishikawa, T. \& Isobe, M. First Asymmetric Total Synthesis of Tetrodotoxin. J. Am. Chem. Soc. 125, 8798-8805 (2003).

8. Hinman, A. \& Du Bois, J. A Stereoselective Synthesis of (-)-Tetrodotoxin. J. Am. Chem. Soc. 125, 11510-11511 (2003).

9. Nishikawa, T., Urabe, D. \& Isobe, M. An Efficient Total Synthesis of Optically Active Tetrodotoxin. Angewandte Chemie International Edition 43, 4782-4785 (2004). 
10. Urabe, D., Nishikawa, T. \& Isobe, M. An Efficient Total Synthesis of Optically Active Tetrodotoxin from Levoglucosenone. Chemistry - An Asian Journal 1, 125-135 (2006).

11. Sato, K. et al. Novel and Stereocontrolled Synthesis of ( \pm )-Tetrodotoxin from myoInositol. J. Org. Chem. 70, 7496-7504 (2005).

12. Sato, K. et al. Stereoselective and Efficient Total Synthesis of Optically Active Tetrodotoxin from d-Glucose. J. Org. Chem. 73, 1234-1242 (2008).

13. Akai, S. et al. Total Synthesis of (-)-Tetrodotoxin from D-Glucose: A New Route to Multi-Functionalized Cyclitol Employing the Ferrier(II) Reaction toward (-)Tetrodotoxin. BCSJ 83, 279-287 (2010).

14. Maehara, T., Motoyama, K., Toma, T., Yokoshima, S. \& Fukuyama, T. Total Synthesis of (-)-Tetrodotoxin and 11-norTTX-6(R)-ol. Angewandte Chemie International Edition 56, 1549-1552 (2017).

15. Murakami, K., Toma, T., Fukuyama, T. \& Yokoshima, S. Total Synthesis of Tetrodotoxin. Angewandte Chemie International Edition 59, 6253-6257 (2020).

16. Cagide-Fagín, F. \& Alonso, R. A Cascade Annulation Based Convergent Approach to Racemic Tetrodotoxin. European Journal of Organic Chemistry 2010, 6741-6747 (2010).

17. Lago-Santomé, H., Meana-Pañeda, R. \& Alonso, R. A Convergent Approach to the Dioxaadamantane Core of ( \pm )-Tetrodotoxin. J. Org. Chem. 79, 4300-4305 (2014).

18. Mendelsohn, B. A. \& Ciufolini, M. A. Approach to Tetrodotoxin via the Oxidative Amidation of a Phenol. Org. Lett. 11, 4736-4739 (2009). 
19. Chau, J., Xu, S. \& Ciufolini, M. A. Assembly of a Key Dienic Intermediate for Tetrodotoxin via a Machetti-DeSarlo Reaction. J. Org. Chem. 78, 11901-11910 (2013).

20. Xu, S. \& Ciufolini, M. A. Formal Synthesis of $( \pm)$-Tetrodotoxin via the Oxidative Amidation of a Phenol: On the Structure of the Sato Lactone. Org. Lett. 17, 24242427 (2015).

21. Baidilov, D. et al. Chemoenzymatic Synthesis of Advanced Intermediates for Formal Total Syntheses of Tetrodotoxin. Angewandte Chemie International Edition 57, 10994-10998 (2018).

22. Keana, J. F. W. \& Kim, C. U. Synthetic intermediates potentially useful for the synthesis of tetrodotoxin and derivatives. III. Synthesis of a key lactone intermediate from shikimic acid. J. Org. Chem. 36, 118-127 (1971).

23. Keana, J. F. W. et al. Synthetic intermediates potentially useful for the synthesis of tetrodotoxin and derivatives. 8. A series of highly functionalized pyrimidinones. $J$. Org. Chem. 48, 3621-3626 (1983).

24. Keana, J. F. W. et al. Synthetic intermediates potentially useful for the synthesis of tetrodotoxin and derivatives. 9. Hydroquinazolines possessing the carbon skeleton of tetrodotoxin. J. Org. Chem. 48, 3627-3631 (1983).

25. Burgey, C. S., Vollerthun, R. \& Fraser-Reid, B. Armed/Disarmed Effects and Adamantyl Expansion of Some Caged Tricyclic Acetals en Route to Tetrodotoxin1,2. J. Org. Chem. 61, 1609-1618 (1996). 
26. Noya, B. \& Alonso, R. Radical cyclisation onto C-3 of 1,6-anhydro- $\beta$-dmannopyranose derivatives. Application to the formation of the C8a centre of (-)tetrodotoxin. Tetrahedron Letters 38, 2745-2748 (1997).

27. Noya, B., Paredes, M. D., Ozores, L. \& Alonso, R. 5-exo Radical Cyclization onto 3Alkoxyketimino-1,6-anhydromannopyranoses. Efficient Preparation of Synthetic Intermediates for (-)-Tetrodotoxin. J. Org. Chem. 65, 5960-5968 (2000).

28. Itoh, T., Watanabe, M. \& Fukuyama, T. Synthetic Approach to Tetrodotoxin. Synlett 2002, 1323-1325 (2002).

29. Ohtani, Y., Shinada, T. \& Ohfune, Y. Stereoselective Construction of a Contiguous Tetraol System in Tetrodotoxin by Means of Repetitive Operations Involving Epoxidation and Ring-opening Reactions of Allyl Sulfone. Synlett 2003, 619-622 (2003).

30. Taber, D. F. \& Storck, P. H. Synthesis of (-)-Tetrodotoxin: Preparation of an Advanced Cyclohexenone Intermediate. J. Org. Chem. 68, 7768-7771 (2003).

31. Manabe, A., Ohfune, Y. \& Shinada, T. Toward the total synthesis of tetrodotoxin: stereoselective construction of the 7-oxanorbornane intermediate. Tetrahedron Letters 55, 6077-6080 (2014).

32. Good, S. N., Sharpe, R. J. \& Johnson, J. S. Highly Functionalized Tricyclic Oxazinanones via Pairwise Oxidative Dearomatization and N-Hydroxycarbamate Dehydrogenation: Molecular Diversity Inspired by Tetrodotoxin. J. Am. Chem. Soc. $139,12422-12425$ (2017). 
33. Bergeron, R. J. \& McManis, J. S. Total synthesis of (.+-.)-15-deoxyspergualin. J. Org. Chem. 52, 1700-1703 (1987).

34. Konrad, D. B., Kicin, B. \& Trauner, D. Concise Asymmetric Synthesis of Kweichowenol A. Synlett 30, 383-386 (2019).

35. Eames, J. et al. An efficient protocol for Sharpless-style racemic dihydroxylation. J. Chem. Soc., Perkin Trans. 1 1095-1104 (1999) doi:10.1039/A900277D.

36. Soengas, R. G. \& Silva, A. M. S. Domino Reaction of lodoglycosides: Synthesis of Carbohydrate-Based Nitroalkenes. European Journal of Organic Chemistry 2013, 5022-5027 (2013).

37. Mukaiyama, T. \& Hoshino, T. The Reactions of Primary Nitroparaffins with Isocyanates1. J. Am. Chem. Soc. 82, 5339-5342 (1960).

38. Hager, A., Vrielink, N., Hager, D., Lefranc, J. \& Trauner, D. Synthetic approaches towards alkaloids bearing a-tertiary amines. Nat. Prod. Rep. 33, 491-522 (2016).

39. Basel, Y. \& Hassner, A. An Improved Method for Preparation of Nitrile Oxides from Nitroalkanes for In Situ Dipolar Cycloadditions. Synthesis 1997, 309-312 (1997).

40. Diethelm, S. \& Carreira, E. M. Total Synthesis of Gelsemoxonine through a Spirocyclopropane Isoxazolidine Ring Contraction. J. Am. Chem. Soc. 137, 60846096 (2015).

41. Cavedon, C. et al. Visible-Light-Mediated Oxidative Debenzylation Enables the Use of Benzyl Ethers as Temporary Protecting Groups. Org. Lett. 23, 514-518 (2021). 
42. Trost, B. M. \& Rhee, Y. H. Ruthenium-Catalyzed Cycloisomerization-Oxidation of Homopropargyl Alcohols. A New Access to Y-Butyrolactones. J. Am. Chem. Soc. 121, 11680-11683 (1999).

43. McDonald, F. E., Reddy, K. S. \& Díaz, Y. Stereoselective Glycosylations of a Family of 6-Deoxy-1,2-glycals Generated by Catalytic Alkynol Cycloisomerization. J. Am. Chem. Soc. 122, 4304-4309 (2000).

44. Zacuto, M. J., Tomita, D., Pirzada, Z. \& Xu, F. Chemoselectivity of the Ru-Catalyzed Cycloisomerization Reaction for the Synthesis of Dihydropyrans; Application to the Synthesis of I-Forosamine. Org. Lett. 12, 684-687 (2010).

45. Beligny, S., Eibauer, S., Maechling, S. \& Blechert, S. Sequential Catalysis: A Metathesis/Dihydroxylation Sequence. Angewandte Chemie International Edition 45, 1900-1903 (2006).

46. Plietker, B. RuO4-Catalyzed Ketohydroxylation of Olefins. J. Org. Chem. 68, 71237125 (2003).

47. Knight, D. W., Morgan, I. R. \& Proctor, A. J. A simple oxidative procedure for the removal of ruthenium residues from metathesis reaction products. Tetrahedron Letters 51, 638-640 (2010).

48. Tsuda, K. et al. Tetrodotoxin. VII. On the Structures of Tetrodotoxin and its Derivatives. Chemical \& Pharmaceutical Bulletin 12, 1357-1374 (1964).

49. Yotsu-Yamashita, M., Jang, J.-H., Cho, Y., Konoki, K. Optimization for simultaneous analysis of tetrodotoxin, 4-epitetrodotoxin, 4,9-anhydrotetrodotoxin and 5,6,11- 
trideoxytetrodotoxin by hydrophilic interaction liquid chromatography-tandem mass spectrometry, Forensic Toxicol., 29, 61-64, (2011).

50. Nakanishi, K. Terpene trilactones from Gingko biloba: From ancient times to the 21st century. Bioorganic \& Medicinal Chemistry 13, 4987-5000 (2005).

51. Curcino Vieira, I. J. \& Braz-Filho, R. Quassinoids: Structural Diversity, Biological Activity and Synthetic Studies. in Studies in Natural Products Chemistry (ed. Atta-urRahman) vol. 33 433-492 (Elsevier, 2006). 


\section{Graphical Abstract}

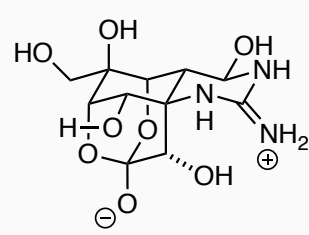

Tetrodotoxin (TTX)

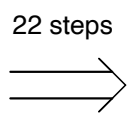

TMS

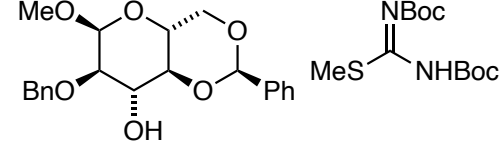

